\title{
Evaluation of PACE4 isoforms as biomarkers in thyroid cancer
}

Laurent Fradet $^{1^{*}}$ (D), Rabia Temmar ${ }^{2}$, Frédéric Couture ${ }^{3}$, Mathieu Belzile ${ }^{1}$, Pierre-Hugues Fortier ${ }^{1}$ and Robert Day ${ }^{3}$

\begin{abstract}
Background: To date, no single molecular marker has been demonstrated as clinically useful in differentiating malignant from benign thyroid nodules when a fine needle aspiration falls in the "unknown significance" categories of the Bethesda Classification. PACE4, a member of the proprotein convertase family of enzymes, has been shown to play a major role in the pathogenesis of prostate cancer, through the formation of an oncogenic isoform named PACE4-altCT. PACE4 isoforms have also been suggested to play a role in other cancers, including thyroid cancer, but have never been investigated in a detailed manner. Our objective is to compare the histochemical distribution of the two major PACE4 isoforms in benign and malignant thyroid nodules, in order to determine their potential usefulness as discriminatory biomarkers.
\end{abstract}

Methods: Thyroid tissues of patients who underwent thyroidectomy were classified according to final pathology. Corresponding tissue sections were immunostained, using two previously validated antibodies raised against the C-terminal end of the two PACE4 isoforms, namely the full-length PACE4 protein (PACE4-FL) and its alternative isoform (PACE4-altCT). Nodules were compared with adjacent normal parenchyma and immunostaining was rated as "low" or "high" by a head and neck pathologist.

Results: Non-lesional thyroid parenchyma did not express PACE4-FL $(p=0.002)$. As a group, malignant $(n=17)$ nodules expressed PACE4-FL significantly more than benign $(n=24)$ nodules (percentage of high immunostaining: $52.9 \%$ vs $4.2 \% ; p=0.001$ ). Reciprocally, there was a statistically lower expression of PACE4-altCT in malignant nodules than in adjacent non-lesional parenchyma $(p=0.014)$. The specificity of a high PACE4-FL immunostaining in determining malignancy was $95.8 \%$ (95\% Cl, $78.9 \%$ to $99.9 \%)$.

Conclusion: This study supports the previously described relationship between PACE4-FL and PACE4-altCT through alternative splicing. It also suggests that PACE4-FL is a promising biomarker for thyroid malignancy. Its high specific expression for malignancy could make it an interesting "rule in" test for thyroid cancer. Further prospective, quantitative studies are currently being designed to address how measurements of PACE4 isoforms could be used in a clinical setting.

Trial registration: This study does not report the results of a health care intervention on human participants. It was nonetheless registered on ClinicalTrials.gov under reference number NCT03160482.

Keywords: Molecular marker, Biomarker, Thyroid nodule, Thyroid Cancer, Fine needle aspiration, Proprotein convertase PACE4

\footnotetext{
* Correspondence: Laurent.Fradet@USherbrooke.ca

${ }^{1}$ Division of Otolaryngology, Department of Surgery, Faculty of Medicine,

Université de Sherbrooke, CIUSSS de l'Estrie - CHUS, Hôpital Hôtel-Dieu de

Sherbrooke, 580 Bowen S, Sherbrooke, QC J1G 2E8, Canada

Full list of author information is available at the end of the article
}

(c) The Author(s). 2018 Open Access This article is distributed under the terms of the Creative Commons Attribution 4.0 International License (http://creativecommons.org/licenses/by/4.0/), which permits unrestricted use, distribution, and reproduction in any medium, provided you give appropriate credit to the original author(s) and the source, provide a link to the Creative Commons license, and indicate if changes were made. The Creative Commons Public Domain Dedication waiver (http://creativecommons.org/publicdomain/zero/1.0/) applies to the data made available in this article, unless otherwise stated. 


\section{Background}

It is estimated that up to $68 \%$ of the general population present at least one detectable thyroid nodule on ultrasound [1]. Current investigation guidelines, published by the American Thyroid Association in 2015, are based on sonographic characterisation of the nodules, followed by fine needle aspiration, depending on their size and features. Cytopathology results are then reported according to the Bethesda Classification, providing a diagnostic category, along with its estimated risk of malignancy. However, only 55 to $74 \%$ of analyzed nodules will be interpreted by cytopathologists as definitively benign and 2 to $5 \%$ as definitively malignant [2]. Remaining nodules will fall in a category of "unknown significance": 2 to $18 \%$ of samples will be categorized as "Atypia of unknown significance - Follicular lesion of unknown significance" (AUS/FLUS; risk of malignancy, $6-18 \%$ [3]), 2 to $25 \%$ as "Follicular neoplasm - Suspicion of follicular neoplasm" (FN/SFN; risk of malignancy, 10-40\% [3]), and 1 to $6 \%$ as "Suspicious for malignancy" (SUSP; risk of malignancy, $45-60 \%$ [3]) [2]. A majority of these patients will end up being operated, despite the relatively low malignancy risk of these categories [4]. The cost of a total thyroidectomy is estimated to about 6000 USD [5], and the potential morbidity related to this surgery is significant [6].

In recent years, researchers have been working on molecular markers that could help to differentiate malignant from benign thyroid nodules when a fine needle aspiration falls in these "unknown significance" categories. However, as stated by the American Thyroid Association, "there is currently no single optimal molecular test that can definitively rule in or rule out malignancy in all cases of indeterminate cytology, and long-term outcome data proving clinical utility [of commercialized biomarkers] are needed" [2]. This justifies the search for new biomarkers.

Paired amino acids converting enzyme 4 (PACE4) is a member of the proprotein convertase family of enzymes. A growing number of publications highlight the role of this protease in carcinogenesis and tumor progression [7-9]. PACE4 plays a role in the oncogenesis of prostate, ovary, and breast cancer [10-12]. More recently, two PACE4 isoforms have been described by Couture et al.: a full-length isoform, PACE4-FL, and its alternative isoform, PACE4-altCT, which is different from its parent isoform in term of autocatalytic processing and cellular trafficking, as it remains inside the secretory pathway without being secreted. The difference between these two isoforms results from an alternative splicing of the PACE4 transcript [13]. In prostate cancer, the PACE4-altCT isoform was shown to be oncogenic. In addition, preliminary data also suggest that this splicing event can also be found in other tissues, including thyroid, and thus could be involved in cancer related events [13].
The objective of this study is to describe the expression of the two PACE4 isoforms among benign and malignant thyroid nodules, in order to determine their value as potential molecular markers for thyroid cancer.

\section{Methods \\ Study design}

This pilot study was designed as a transversal, descriptive study. Our goal is to describe the expression profile of PACE4 isoforms among thyroid nodules and adjacent normal parenchyma in order to justify further prospective, quantitative studies on this proprotein, that could lead to clinical applications.

\section{Ethics approval}

This research protocol was presented to the Institution Ethic Board (CIUSSS de l'Estrie - CHUS; certified FWA $\# 00005894$ and IRB00003849) and granted with full approval.

\section{Patients samples}

A list of all patients who underwent either total thyroidectomy or thyroid lobectomy at the CIUSSS de l'Estrie CHUS, from January 2014 to May 2016, was prepared by the medical archives.

These 243 medical files were reviewed by the main investigator (Fig. 1). Patients were classified based on final pathological diagnosis. We chose to study the most common variants of well-differentiated thyroid cancer, i.e. papillary carcinoma (specifically, both its classical and follicular variants) and follicular carcinoma. For benign nodules, we also chose to study the most frequently encountered types, i.e. hyperplasic, colloid and adenomatous nodules, and follicular adenoma. Recruitment was continued randomly until a total of 5 patients per diagnostic category was reached. We estimated that this number of patients per category would be sufficient to observe trends in results. We added to this cohort two cases of medullary cancer that were identified in our database and two cases of lymphocytic thyroiditis, along with two more cases of follicular adenoma that were previously classified as "oncocytic nodules", for a total of 41 patients (Fig. 1). Revision of the final pathology led to three colloid nodules being reclassified as hyperplastic nodules. As such, only two sample were analysed for the colloid nodule category and eight fell in the hyperplastic nodule category (Fig. 1).

Patients were contacted by the main investigator (LF) for consent. Upon consent, pathological slides of each patient were reviewed by a head and neck pathologist (RT) to confirm the diagnosis. In particular, follicular variants of papillary carcinoma were thoroughly reviewed to make sure they were not reclassified as the newly described variant of Non-invasive Follicular Thyroid Neoplasm with Papillary-like 


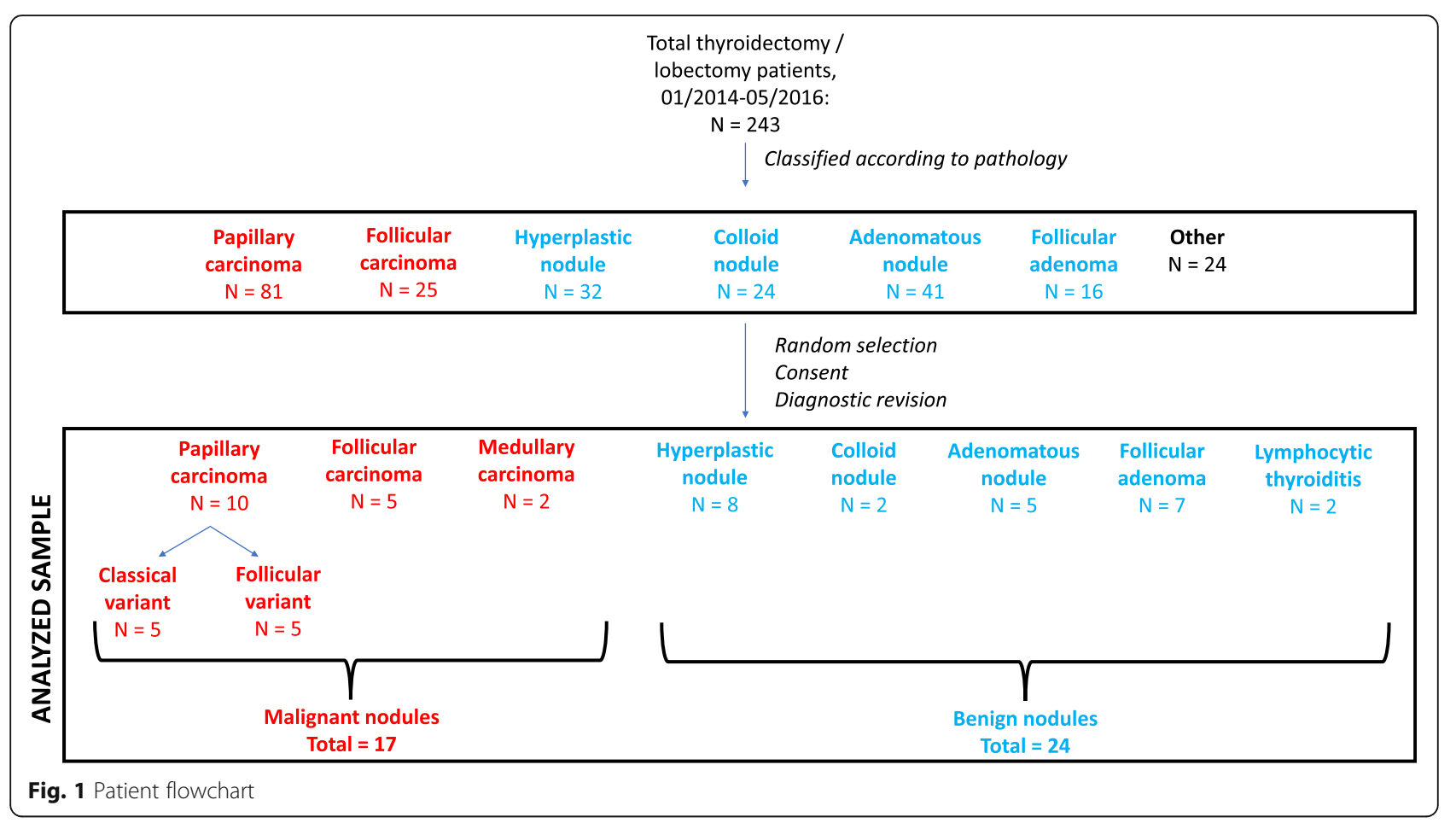

Nuclear Features (NIFTP), which is considered a benign neoplasm. New $4 \mu \mathrm{m}$-thick slides were cut from paraffin blocs and were used to perform the immunohistochemistry technique. Blocks were specifically chosen by the pathologist to ensure that both lesional (nodule) and non-lesional (adjacent normal) thyroid parenchyma would be found on each slide, in order to compare the expression of PACE4 in these two components.

\section{Immunohistochemistry technique}

Rabbit polyclonal immunoglobulins targeting either PACE4-FL or PACE4-altCT were purified on an antigenic peptide-coated chromatographic column. The sensitivity and specificity of these antibodies was previously demonstrated [13].

Using these antibodies, an automatized immunohistochemistry technique was performed with a Dako device (Agilent technologies, Santa Clara, CA). Slides were stained with a horseradish-peroxidase reaction and counterstained with Harris hematoxylin (Sigma-Aldrich, St-Louis, Missouri). Detailed description of this technique was previously published by Couture et al. [13].

Before performing this technique on our recruited patient sample, validation of the immunohistochemistry method was undertaken using four slides of thyroid tissues that included both non-lesional parenchyma and specific types of nodules. This enabled us to confirm that the thyroid tissue stained properly with this technique.

\section{Data acquisition}

Immunostained slides were interpreted by a specialized head and neck pathologist (RT). The intensity of immunostaining was described as either "low" (for no to slight staining) or "high" (for moderate to intense staining), both for the studied nodule (i.e. lesional parenchyma, either cancerous or benign) and for adjacent non-lesional (i.e. normal) parenchyma. Captions of representative fields were taken with a microscope digital camera (Olympus DP26; Olympus, Tokyo, Japan), under 20X magnification.

\section{Statistical analysis}

Statistical analyses were independently run and validated by the institution biostatistician.

Wilson's 95\% confidence intervals were calculated on the percentage of high immunostaining for every diagnosis.

Comparison of the percentage of high immunostaining between lesional and non-lesional parenchyma, for each antibody, was made with the McNemar test on two by two contingence tables using SAS Software, version 9.3 (SAS Corporation, Cary, NC).

Comparison of the percentage of high immunostaining between cancerous and benign nodules, for each antibody, was made with the Fischer exact test on two by two contingence tables using SPSS Software, version 25 (IBM, Armonk, NY). Results from multiples comparisons between each pair of diagnosis were obtained by Fisher exact tests and $p$-values were corrected by the false discovery rate method, as described by Benjamini and Hochberg. 
The sensitivity and specificity of each PACE4 isoform, with $95 \%$ confidence intervals, were determined on two by two contingence tables using the MedCalc online software (www.medcalc.com).

\section{Results}

\section{Data spreadsheet}

The original data spreadsheet is available as Additional file 1.

\section{Expression of PACE4-FL}

For all of the 37 samples in which non-lesional parenchyma was identified, a low PACE4-FL immunostaining was observed (Fig. 2). There was no statistical difference between the expression of PACE4-FL in lesional and non-lesional parenchyma for benign lesions, which both exhibited a low expression of PACE4-FL $(p=0.317$; Fig. 2). However, the expression of PACE4-FL was statistically higher in lesional than in non-lesional parenchyma for malignant lesions ( $p=0.003$; Figs. 2 and 3).

As a group, malignant nodules expressed PACE4-FL to levels significantly higher than benign nodules (percentage of high immunostaining: $52.9 \%$ vs $4.2 \% ; p=0.001$; Fig. 2). Multiple comparison analyses of the proportion of high immunostaining among the different nodule types can be found in Additional file 2. The sensitivity and specificity of a high PACE4-FL immunostaining in determining malignancy were respectively 52.9\% (95\% CI, $27.81 \%$ to $77.02 \%$ ) and $95.8 \%$ (95\% CI, $78.9 \%$ to 99.9\%). Figure 4 presents the percentage of high immunostaining for every nodule type; representative fields are found in Fig. 5 for malignant and in Fig. 6 for benign nodules.
In malignancies, expression of the protein was most pronounced for the classical variant of papillary carcinoma, which displayed an apically-distributed high immunostaining in all five studied samples (Fig. 5a). On the opposite, immunostaining was low for all follicular variant of papillary carcinoma specimens examined (Fig. 5b). For follicular carcinoma, three out of five slides displayed high immunostaining, which was especially pronounced in the more compact, oncocytic zones of the nodules (Fig. 5c, arrow). One medullary carcinoma included in this study showed a high immunostaining (Fig. 5d), while the second displayed a low immunostaining.

All benign nodule types consistently showed no expression of PACE4 (Figs. 2, 4 and 6), except for one follicular adenoma sample.

\section{Expression of PACE4-altCT}

For benign nodules, PACE4-altCT was equally expressed in both lesional and non-lesional thyroid parenchyma $(p=0.739$; Fig. 7). However, for malignant nodules, there was a statistically higher expression of PACE4-altCT in non lesional parenchyma, compared to lesional parenchyma ( $p=0.014$; Figs. 7 and 8).

As a group, malignant nodules did not express PACE4-altCT significantly more than benign nodules (percentage of high immunostaining: $47.1 \%$ vs $58.2 \% ; p=0.537$; Fig. 8). The sensitivity and specificity of PACE4-altCT for differentiating malignant and benign nodules, based on a high immunostaining, were respectively $47.1 \%$ (95\% CI, $23.0 \%$ to $72.2 \%$ ) and $41.7 \%$ ( $95 \%$ CI, $22.11 \%$ to $63.4 \%$ ). When comparing the percentage of high immunostaining from one type of nodule to the other, no significant trend

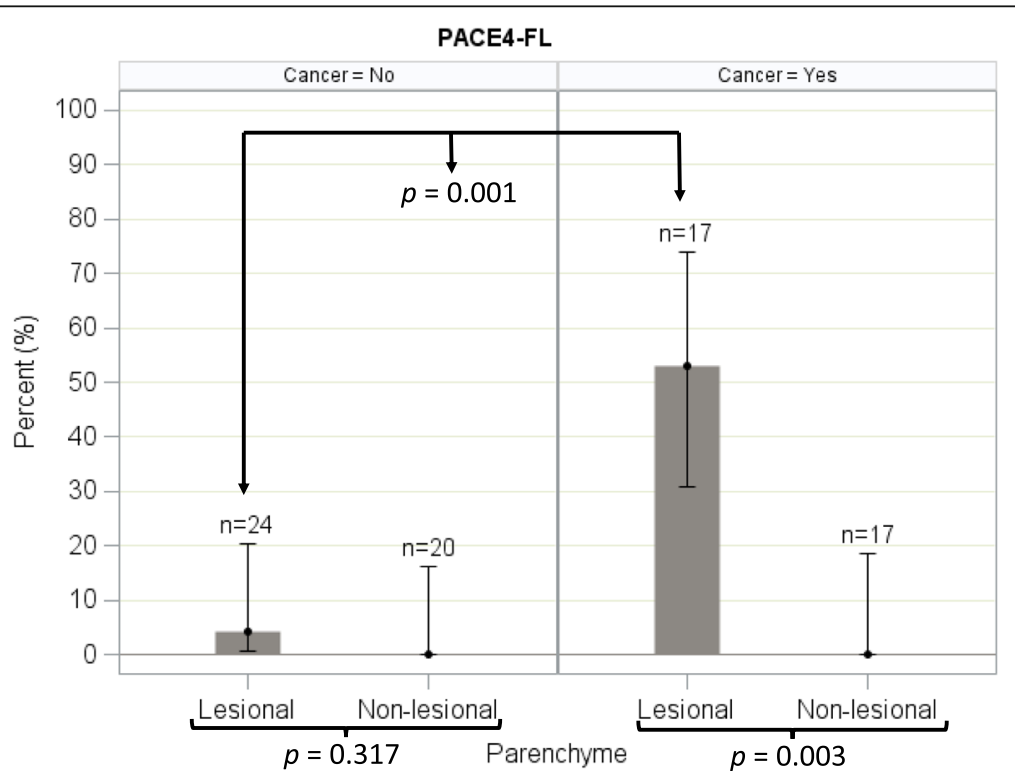

Fig. 2 Proportion of high immunostaining in lesional (nodule) and non-lesional (normal) thyroid parenchyma, PACE4-FL. Error bars represent the Wilson's 95\% confidence intervals 

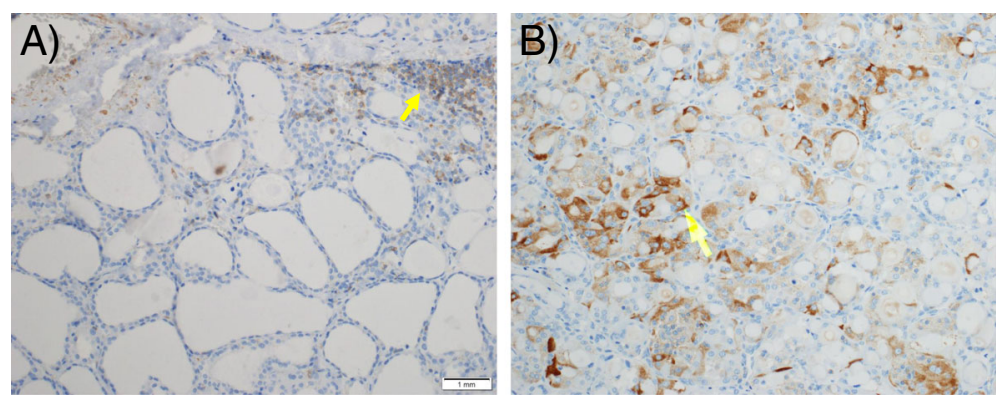

Fig. 3 Comparison of representative fields for a case of follicular carcinoma (malignancy), PACE4-FL, under 20X magnification. a Non-lesional (normal) parenchyma, which did not exhibit staining; the only immunostained cells are lymphocytes (arrow). b Lesional parenchyma (nodule), demonstrating high immunostaining (arrow)

could be noted (Fig. 9; Additional file 2). While the heterogeneity of results make it difficult to define representative fields, Fig. 10 presents some examples.

\section{Discussion}

\section{The burden of thyroid cancer and the limitations of cytology}

In the last decades, the incidence of thyroid cancer has more than tripled [14]. Following this tendancy, in 2019, papillary thyroid cancer would become be the third most prevalent cancer among women in the United States, with associated annual costs estimated between 19 and 21 billion dollars [15]. This increase is mainly due to "small papillary carcinomas", and is probably attributable to increased sreening and the more widespread use of ultrasound imaging [16].
As thyroid cancer is an indolent disease, the typical clinical presentation is that of an incidentally detected thyroid nodule, either on physical or radiologic examination [2]. However, only 7 to $15 \%$ of thyroid nodules will prove to be malignant [2]. The diagnostic workup of these nodules is largely limited by the poor performance of cytology in providing a definitive diagnosis in a high percentage of cases.

The Bethesda Cytology Classification, recently updated [3], enables researchers, pathologists, and clinicians to use a common language when it comes to thyroid cytopathology. While the malignancy risks related to the benign and malignant categories are clear, categories of "unknown significance" represent a clinical dilema to the physician.

Commercialized biomarker assays, designed with the intent of clarifying the risk of malignancy for these cases,

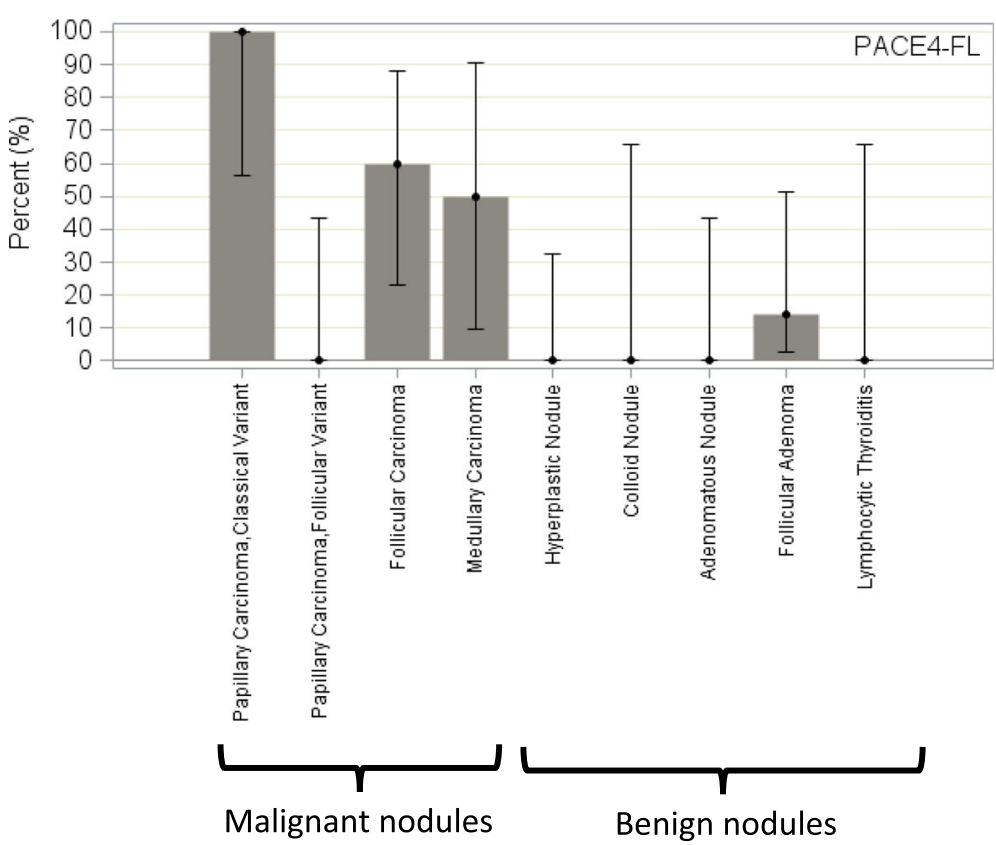

Fig. 4 Percentage of high immunostaining for every nodule type (lesional parenchyma), PACE4-FL. Error bars represent the Wilson's 95\% confidence intervals 

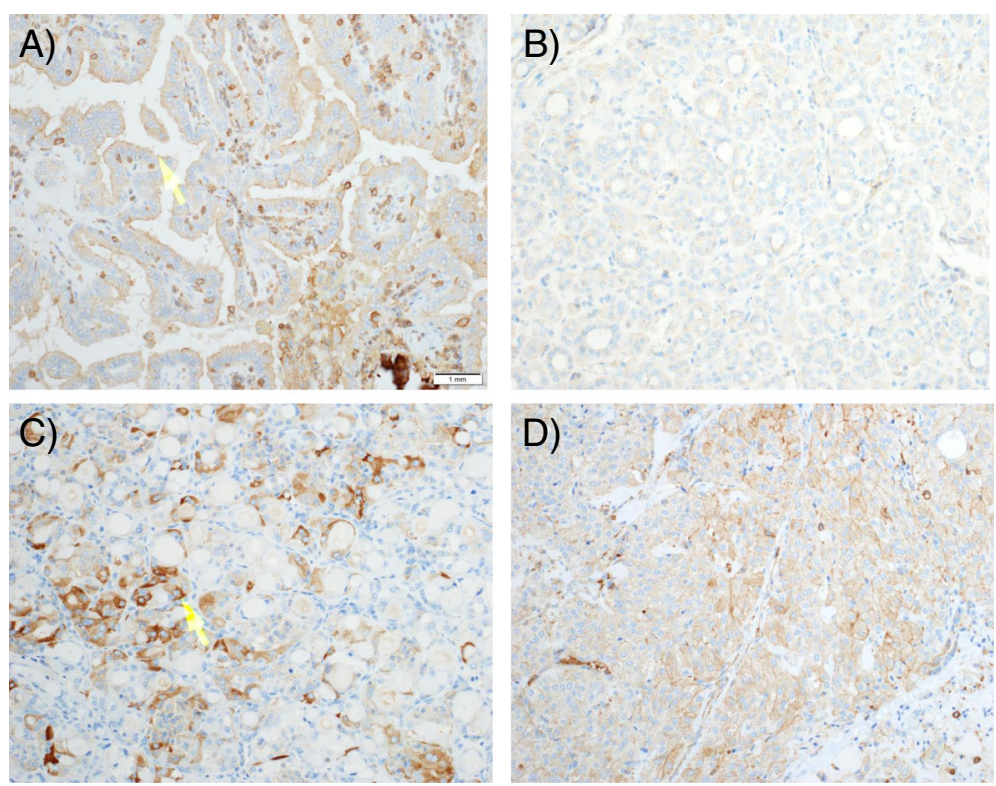

Fig. 5 Representative fields for malignant nodules, PACE4-FL, magnification 20X. a Papillary carcinoma, classical variant (high immunostaining; arrow). b Papillary carcinoma, follicular variant (low immunostaining). c Follicular carcinoma (high immunostaining; arrow). d Medullary carcinoma (high immunostaining)

are both limited in their sensitivity or specificity and expensive. For example, the PCR mutational analysis of 7 genes commercialised under the name ThyroSeq (CBLPath, Rye Brook, NY) has a specificity of 99\%, but its sensivity varies from 44 to $100 \%$ [2], depending on studies. The galactine- 3 immunohistochemistry technique published by Bartolazzi et al. also has a high specificity (93\%), but a lower sensitivity (78\%) [2]. The 167 gene expression classifier described by Alexander et al., commercialized under the name Afirma (Veracyte, San Francisco, CA), has a 96\% sensitivity, but a 31\% specificity [17]. Finally, combination of the ThyGenX and ThyraMir tests (Interspace Diagnostics, Parsippany, NJ) has a sensitivity of $89 \%$ and a specificity of $85 \%$ [18].
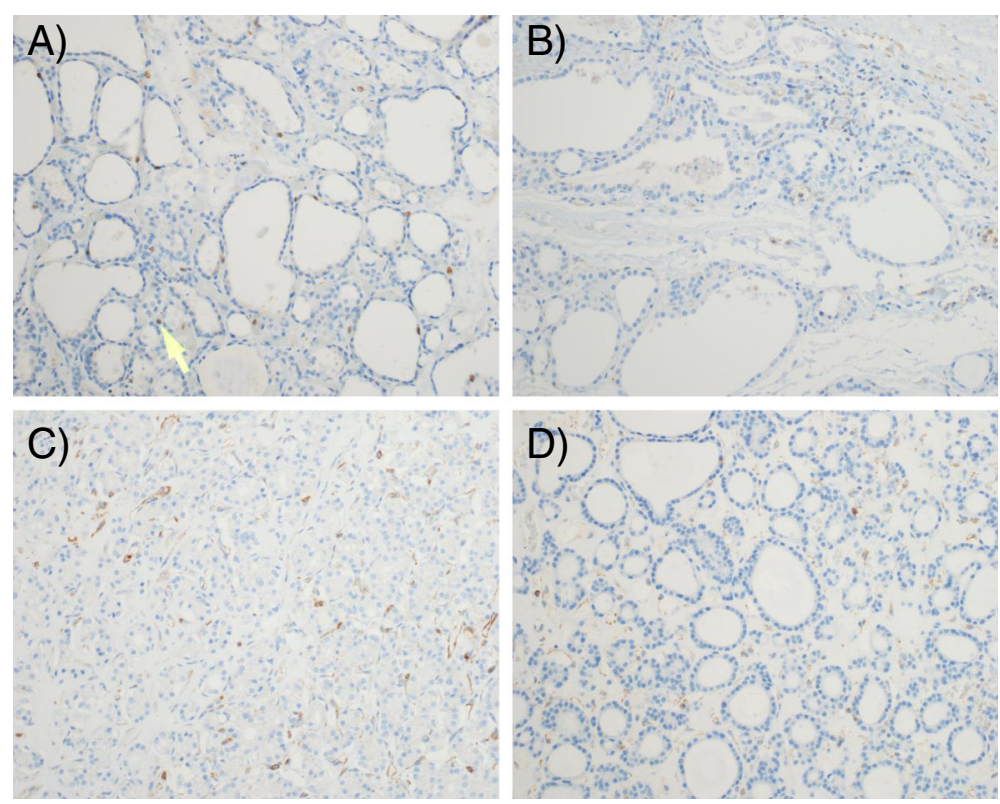

Fig. 6 Representative fields for benign nodules, PACE4-FL, magnification 20X. a Hyperplastic nodule; the arrow indicates an immunostaining lymphocyte. b Colloid nodule. c Adenomatous nodule. d Follicular adenoma 


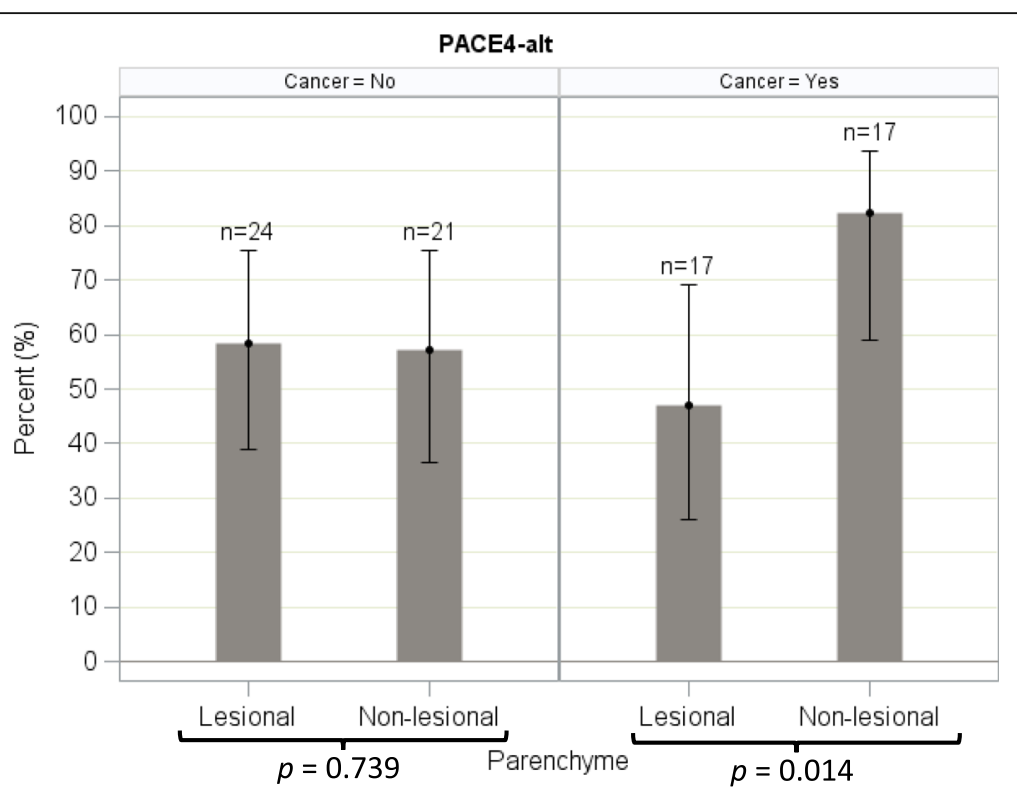

Fig. 7 Proportion of high immunostaining in lesional (nodule) and non-lesional (normal) thyroid parenchyma, PACE4-altCT. Error bars represent the Wilson's 95\% confidence intervals

\section{PACE4 as a biomarker}

The family of proprotein convertases is a group of nine enzymes, composed of PC1/3, PC2, furin, PC4, PC 5/6, PACE4, PC7, SKI-1/S1P, and PCSK9. Enzymes from this family, especially furin and PACE4, have been investigated regarding their implications in tumor progression, since they are involved in the processing and activation of various protein precursors that have been associated with cancer progression, including tumor growth factor beta (TGF $\beta)$, matrix metalloproteinases (MMP), and insulin-like growth factor receptors (IGF1 R) [7].

It has been demonstrated that, among various cell lineages, overexpression of PACE4 confers oncogenic growth-sustaining functions $[19,20]$. The central role of PACE4 in the sustained growth capabilities of prostate cancer cells has been demonstrated by the induction of proliferation arrest using either a PACE4 inhibitor or PACE4-specific gene-silencing tools [21]. Similar results have been obtained for breast cancer [12]. In recent work, Couture et al. characterized a novel
PACE4 isoform, PACE4-altCT, that is generated by an alternative splicing mechanism, and is oncogenic in prostate cancer cells [13]. Also included in this study was a preliminary scan of numerous cancer tissues at the mRNA level to examine PACE4-FL and PACE4-altCT, which suggested that changes in splicing ratios could be indicative of oncogenic status.

PACE4 expression is known to be inconsistent among tissues. The Human Protein Atlas [22] and another previous study [23] suggested that expression levels in thyroid tissues are relatively low. To our knowledge, no study had focused until now on the expression pattern of PACE4 among different thyroid nodule types. Moreover, no previous study had investigated the full-length and alternative isoforms of PACE4 in thyroid tissues.

\section{PACE4-FL: A potential biomarker for malignancy}

A good biomarker would need to be consistently expressed in malignant nodules, and absent of both

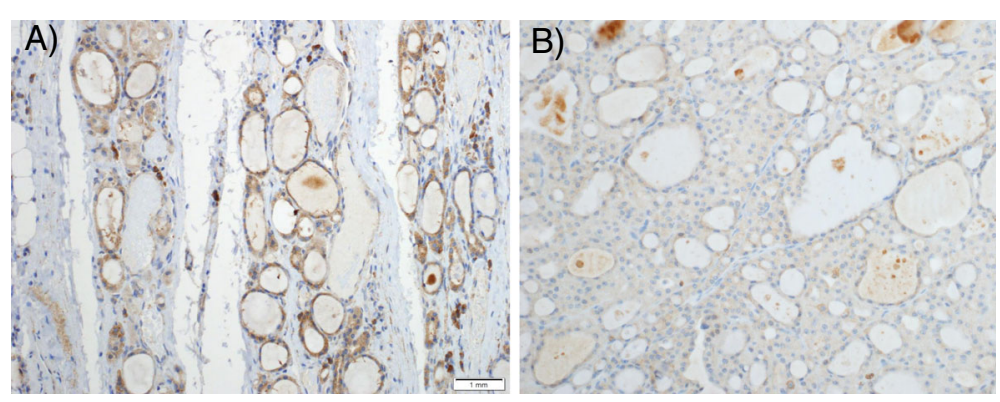

Fig. 8 Comparison of representative fields for a case of follicular carcinoma (malignancy), PACE4-altCT, 20X magnification. a Non-lesional (normal) parenchyma (high immunostaining). b Lesional (nodule) parenchyma (low immunostaining) 


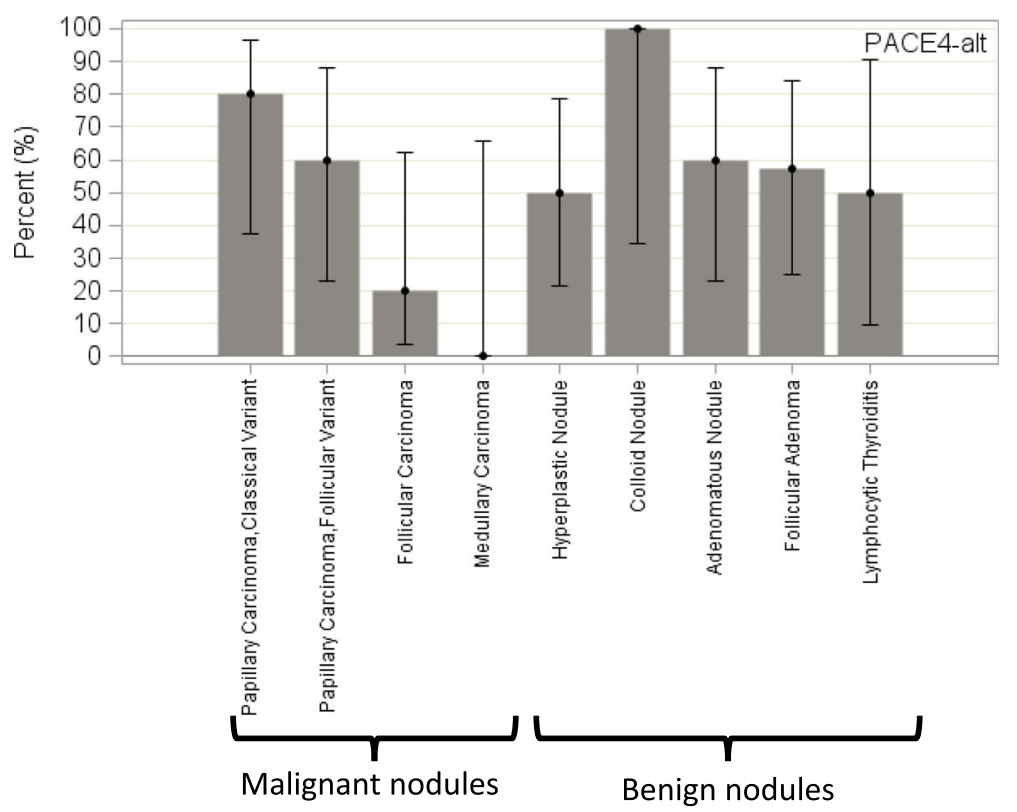

Fig. 9 Percentage of high immunostaining among every nodule type (lesional parenchyma), PACE4-altCT. Error bars represent the Wilson's 95\% confidence intervals
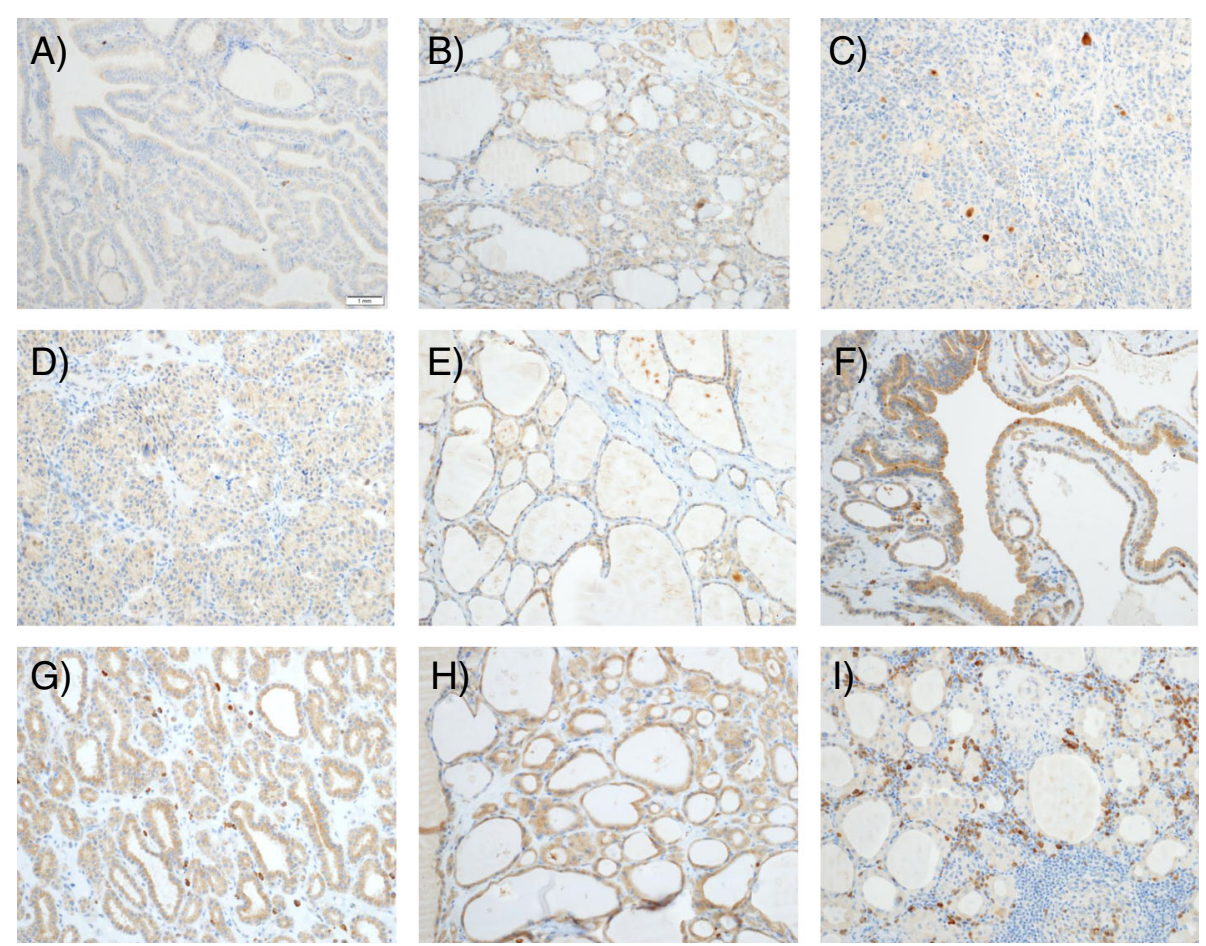

Fig. 10 Representative fields, PACE4-altCT, 20X magnification. a Papillary carcinoma, classical variant. b Papillary carcinoma, follicular variant. c Follicular carcinoma. $\mathbf{d}$ Medullary carcinoma. e Hyperplastic nodule. $\mathbf{f}$ Colloid nodule. $\mathbf{g}$ Adenomatous nodule. $\mathbf{h}$ Follicular adenoma. i Lymphocytic thyroiditis 
non-lesional (normal) parenchyma and benign thyroid nodules. Our data suggest that PACE4-FL is not expressed in non-lesional thyroid parenchyma and in benign nodules and thus, has good specificity (95.8\%; 95\% CI: 78.8-99.9\%) for malignancy. Hence, presence of this isoform could be a good "rule in" test for cancer.

On the other hand, the limited sensitivity of PACE4-FL for malignancy $(52.9 \%$; $95 \% \mathrm{CI}, 27.81 \%$ to $77.02 \%)$ is mostly attributable to false negative results consistently obtained with the follicular variant of papillary carcinoma. Indeed, all of the follicular variant of papillary carcinoma samples and two out of five follicular carcinoma samples presented a low immunostaining. The high proportion of follicular malignancies selected in our study, due to its design, in comparison with its lower incidence in real-life cohorts, explain the low sensitivity of PACE4-FL that we report.

It is well established that in cytopathology, "follicular-type" lesions are the most difficult to evaluate. This difficulty is also encountered with some mutational panel biomarker assays. For example, in a multi-institutional, double-blinded study evaluating the performance of the molecular analysis of 17 oncogenic alterations in nodules, Beaudenon-Huibregtse et al. reported that 8 of the 14 false-negative results proved to be follicular variants of papillary carcinomas on final pathology [24]. While 4 of these were noninvasive and encapsulated, and thus would nowadays correspond to benign nodules of the NIFTP category, the other 4 showed invasive characteristics, and thus would still be considered malignant with updated criteria [24]. Nikiforov et al., in their prospective mutational panel analysis of 1056 fine needle aspiration samples, reported that $13 \%$ of their false-negative results were follicular carcinomas on final pathology, and $62 \%$ were noninvasive encapsulated follicular variants of papillary carcinoma, which would be considered benign nowadays [25].

Two hypothesis can be proposed to explain false-negative results for follicular variants of papillary carcinomas. First, genetic mutations from one subtype of thyroid cancer to the other are quite variable and characteristic [26]. For example, the BRAF V600E mutation is strongly associated with conventional papillary carcinoma, BRAF K601E, with the follicular variant of papillary carcinoma, and PTEN, with follicular carcinoma [26]. As such, it is possible that PACE4-FL is in fact strongly correlated to the classical variant of papillary carcinoma, but less so with "follicular-type" malignancies. Second, it is well described that some mutations are strongly correlated with the aggressiveness or other specific clinical features of the thyroid cancer. For example, the PAX8/PPARY rearrangement is linked, among others, to vascular invasion [27], while the $p 53$ mutation is linked to tumor dedifferentiation [26]. While the limited number of specimens included in this study precludes us to do so, further studies could explore if PACE4 is linked to a specific phenotype or clinical feature of thyroid cancer, and thus would rather be a marker of this phenotype. This might also be one path of explanation as to why the expression was varying among some nodule types, as for the medullar carcinomas.

Finally, as to the histochemical distribution, it is interesting to mention that the apical positivity of PACE4-FL, particularly marked for papillary carcinoma (Fig. 5a), is consistent with data by Couture et al. [13] and Nour et al. [28], suggesting that PACE4-FL is readily reaching the cell surface and accumulating in the extracellular matrix.

\section{PACE4-altCT: A demonstration of the relationship between PACE4-FL and PACE4-altCT in alternative splicing}

As opposed to PACE4-FL, PACE4-altCT did not prove to be either sensitive nor specific for malignancy. However, while PACE4-FL is more expressed in cancer than in adjacent normal parenchyma, the opposite is true for PACE4-altCT: a statistically significant reduction in PACE4-altCT was noted in malignant nodules when compared to adjacent non-lesional parenchyma (Fig. 7).

As such, the present study on PACE4 expression in thyroid cancer presents a contrast with data obtained in previous work on prostate cancer. In prostate cancer, there is a clear shift in alternative splicing to favour PACE-altCT [13], while in the present study, the inverse appears to be true. While our data support the tight relationship between these two isoforms, it also suggests that in thyroid carcinoma, different mechanisms are affecting the alternative splicing events. In prostate cancer, increased PACE4-altCT was shown to be due to hypomethylating epigenetic events that favour PACE4-altCT formation [13]. Although this was not verified in the present study, it is possible that increased DNA methylation is occurring in thyroid cancer, leading to alternative splicing events that favour PACE4-FL. Indeed, DNA methylation signatures have been examined and shown to have specific patterns in various thyroid subtypes [29]. However, since our study only focussed on PACE4 isoforms analysis in terms of proteins without examining RNA levels nor splicing activities, further studies will be needed to determine if PACE4-FL increases are due to gene overexpression or splicing regulation. Correlation with methylation signatures would also provide a complete picture of how PACE4 is involved in thyroid carcinoma, as previously done for other cancer types [13].

\section{Limitations of the study}

- The number of samples per thyroid pathology is limited, although statistical significance was obtained. A larger sampling would be of interest and may allow to identify additional statistically significant differences between the various pathologies, owing to a higher study power. 
- Immunohistochemistry is a semi-quantitative method, with inherent interobserver variability and subjectivity. While quantitative results could have been obtained with immunofluorescence, no immunofluorescence method have been described as of today for PACE4.

\section{Further studies}

A follow-up study, using quantitative Polymerase Chain Reaction (qPCR) to determine the quantitative expression levels as well as the splicing indexes of PACE4 among thyroid cytology specimens, is currently being designed to determine the clinical usefulness of PACE4 as a biomarker. Studies to correlate methylation signature and PACE4 alternative splicing in thyroid cancer subtypes would also be highly informative. Further studies could also investigate the yield of a combination of PACE4-FL to other described biomarkers more specific for follicular lesion. Finally, as there is currently a lack of targeted therapy in thyroid cancer, especially for non-iodine-avid lesions [26], and as an inhibitor of PACE4 has already been developed and tested in cellulo for various cancer types [30], PACE4 could eventually be explored as an oncologic target in thyroid cancer.

\section{Conclusion}

This study is the first of its kind to explore the expression of PACE4, in both its full-length and alternative isoforms, in lesional and non-lesional thyroid parenchyma, using previously validated antibodies and an automatized immunohistochemistry technique. Our results suggest that PACE4-FL is not constitutionally expressed in normal thyroid tissue, and that its expression is highly specific to malignancy, making it a potential "rule in" test for cancer. PACE4-altCT displayed the opposite relationship, being less expressed in malignant thyroid nodules than in non-lesional thyroid parenchyma. This relationship is consistent with the alternative splicing mechanism of PACE4 and suggests that increased methylation may be occurring in thyroid cancer. This study justifies further research on the subject, with the intent of defining more precisely the role of PACE4 as a biomarker for thyroid malignancy and as a potential oncologic target.

\section{Additional files}

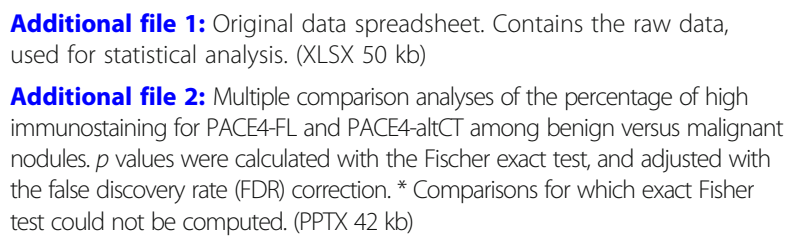

\section{Abbreviations}

FN/SFN: Follicular neoplasm - Suspicion of follicular neoplasm 621; IGF1 R: Insulin-like Growth Factor One Receptor 626; MMP: Matrix

Metalloproteinases 625; NIFTP: Noninvasive Follicular Thyroid Neoplasm with
Papillary-like Nuclear Features; qPCR: Quantitative Polymerase Chain Reaction 623; SUSP: Suspicious for malignancy 622; TGF $\beta$ : Tumor Growth Factor Beta 624; US/FLUS: Atypia of unknown significance - Follicular lesion of unknown significance 620

\section{Acknowledgements}

The authors would like to acknowledge the work of Amélie Boyer, pathology technician, for performing the immunohistochemistries, and of Marie-Pierre Garant, biostatistician, for performing and validating the statistics.

\section{Funding}

This work was supported by grants from the Canadian Cancer Society (grant $\# 701590$ to RD) and Fondation Mon Etoile (to RD). FC is funded by Prostate Cancer Canada (Award \#GS2014-02), by the Canadian Institutes of Health Research (CIHR) Graduate Studentship Award, and by the Banting and Charles Best Canada Graduate Scholarships (grant \#315690). This project was also funded by a research grant awarded by the Department of Surgery of the Université de Sherbrooke to LF, PHF, and MB.

\section{Availability of data and materials}

All data generated or analysed during this study are included in this published article and its supplementary information files (Additional file 1).

\section{Authors' contributions}

LF original research idea, preparation and submission of the research protocol, recruitment of participants, interpretation of the results, redaction of the manuscript. RT revision of the research protocol, selection and revision of the pathology slides, interpretation of immunohistochemistry, revision of the manuscript. RD revision of the research protocol, interpretation of the results, revision of the manuscript. FC revision of the research protocol, interpretation of immunohistochemistry, revision of the manuscript. MB revision of the research protocol, interpretation of the results, revision of the manuscript. PHF revision of the research protocol, interpretation of the results, revision of the manuscript. All authors read and approved the final manuscript.

\section{Authors' information}

LF is a PGY-4 Otolaryngology resident at the Université de Sherbrooke. He has a special interest for head and neck surgery and is currently in the application process of the American Head and Neck Society Fellowship Match. $\mathrm{RT}$ is a head and neck pathologist and cytopathologist at the CIUSSS de l'Estrie - CHUS.

$\mathrm{RD}$ is a Full Professor at the Université de Sherbrooke. His research lab is focused on the role of proprotein convertases with applications in health and disease.

FC is a researcher at TransBlOTech. He is a former PhD student of the Robert Day lab and developed an expertise with proprotein convertases throughout his graduate studies.

MB and PHF are head and neck surgeons at the CIUSSS de l'Estrie - CHUS.

\section{Ethics approval and consent to participate}

This study was approved by our institution's Human Research Ethics Committee (Comité d'éthique de la recherche du CIUSSS de l'Estrie - CHUS), certified under the norms FWA \#00005894 and IRB00003849, under the reference number 2017-1517.

Participants were contacted by the main investigator (LF). Verbal consent was obtained after explaining the project and potential risks encountered. Written consent was subsequently obtained using a form approved by the Ethics Committee and available upon request.

\section{Consent for publication}

Not applicable.

\section{Competing interests}

The authors declare that they have no competing interests.

\section{Publisher's Note}

Springer Nature remains neutral with regard to jurisdictional claims in published maps and institutional affiliations. 


\section{Author details}

'Division of Otolaryngology, Department of Surgery, Faculty of Medicine, Université de Sherbrooke, CIUSSS de l'Estrie - CHUS, Hôpital Hôtel-Dieu de Sherbrooke, 580 Bowen S, Sherbrooke, QC J1G 2E8, Canada. ${ }^{2}$ Department of Pathology, Faculty of Medicine, Université de Sherbrooke, CIUSSS de l'Estrie CHUS, Hôpital Hôtel-Dieu de Sherbrooke, 580 Bowen S, Sherbrooke, QC J1G 2E8, Canada. ${ }^{3}$ Divison of Urology, Departemnt of Surgery, Faculty of Medicine, Université de Sherbrooke, Institut de pharmacologie de Sherbrooke, 3001 12th Ave N, Sherbrooke, QC J1H 5N4, Canada.

Received: 6 April 2018 Accepted: 1 October 2018 Published online: 19 October 2018

\section{References}

1. Guth S, Theune U, Aberle J, Galach A, Bamberger CM. Very high prevalence of thyroid nodules detected by high frequency $(13 \mathrm{MHz})$ ultrasound examination. Eur J Clin Investig. 2009;39:699-706.

2. Haugen BR, Alexander EK, Bible KC, Doherty GM, Mandel SJ, Nikiforov YE, Pacini F, Randolph GW, Sawka AM, Schlumberger M, Schuff KG, Sherman SI, Sosa JA, Steward DL, Tuttle RM, Wartofsky L. American Thyroid Association management guidelines for adult patients with thyroid nodules and differentiated thyroid Cancer: the American Thyroid Association guidelines task force on thyroid nodules and differentiated thyroid Cancer. Thyroid. 2016;26(1):1-133.

3. Cibas ES, Ali SZ. The 2017 Bethesda System for Reporting Thyroid Cytopathology. Thyroid. 2017:27(11):1341-6.

4. Baloch ZW, LiVolsi VA, Asa SL, Rosai J, Merino MJ, Randolph G, Vielh P, DeMay RM, Sidawy MK, Frable WJ. Diagnostic terminology and morphologic criteria for cytologic diagnosis of thyroid lesions: a synopsis of the National Cancer Institute thyroid fine-needle aspiration state of the science conference. Diagn Cytopathol. 2008:36(6):425-37.

5. Takami H, Ito Y, Okamoto T, Onoda N, Noguchi H, Yoshida A. Revisiting the guidelines issued by the Japanese Society of Thyroid Surgeons and Japan Association of endocrine surgeons: a gradual move towards consensus between Japanese and Western practice in the management of thyroid carcinoma. World J Surg. 2014;38:2002-10.

6. Margolick J, Chen W, Wiseman S. Systematic review and meta-analysis of unplanned reoperations, emergency department visits, and hospital readmission after thyroidectomy. Thyroid. 2018 Mar 27; doi: https://doi.org/ 10.1089/thy.2017.0543

7. Khatib AM, Siegfried G, Chrétien M, Metrakos P, Seidah NG. Proprotein convertases in tumor progression and malignancy novel targets in cancer therapy. Am J Pathol. 2002;160(6):1921-35.

8. Bassi DE, Fu J, Lopez de Cicco R, Klein-Szanto AJ. Proprotein convertases: "master switches" in the regulation of tumor growth and progression. Mol Carcinog. 2005:44(3):151-61.

9. Artenstein AW, Opal SM. Proprotein convertases in health and disease. N Engl J Med. 2011;365:2507-18.

10. Couture F, D'Anjou F, Desjardins R, Boudreau F, Day R. Role of proprotein convertases in prostate cancer progression. Neoplasia. 2012;14(11):1032-42.

11. Longuespée R, Couture F, Levesque C, Kwiatkowska A, Desjardins R, Gagnon S, Vergara D, Maffia M, Fournier I, Salzet M, Day R. Implications of Proprotein convertases in ovarian Cancer cell proliferation and tumor progression: insights for PACE4 as a therapeutic target. Transl Oncol. 2014;7(3):410-9.

12. Panet F, Couture F, Kwiatkowska A, Desjardins R, Guérin B, Day R. PACE4 is an important driver of ZR-75-1 estrogen receptor-positive breast cancer proliferation and tumor progression. Eur J Cell Biol. 2017;96(5):469-75.

13. Couture F, Sabbagh R, Kwiatkowska A, Desjardins R, Guay SP, Bouchard L, Day R. PACE4 undergoes an oncogenic alternative splicing switch in Cancer. Cancer Res $2017 ; 15 ; 77(24): 6863-6879$.

14. Davies L, Welch HG. Current thyroid cancer trends in the United States. JAMA Otolaryngol Head Neck Surg. 2014;140:317-22.

15. Aschebrook-Kilfoy B, Schechter RB, Shih YC, Kaplan EL, Chiu BC, Angelos P, Grogan $\mathrm{RH}$. The clinical and economic burden of a sustained increase in thyroid cancer incidence. Cancer Epidemiol Biomark Prev. 2013;22:1252-9.

16. Vaccarella S, Franceschi S, Bray F, Wild CP, Plummer M, Dal Maso L. Worldwide thyroid-Cancer epidemic? The increasing impact of Overdiagnosis. NEJM. 2016;375(7):614-7.

17. Santhanam P, Khthir R, Gress T, Elkadry A, Olajide O, Yaqub A, Driscoll H. Gene expression classifier for the diagnosis of indeterminate thyroid nodules: a meta-analysis. Med Oncol. 2016;33(2):14.
18. Labourier E, Beaudenon A, Wylie D, Giordano TJ. Multi-categorical testing for miRNA, mRNA and DNA on fine needle aspiration improves the preoperative diagnosis of thyroid nodules with indeterminate cytology. ENDO 2015. Presented at the 97th Meeting and Expo of the Endocrine Society March 5-8, 2015. SAT-344.

19. D'Anjou F, Routhier S, Perreault J-P, Latil A, Bonnel D, Fournier I, et al. Molecular validation of PACE4 as a target in prostate cancer. Transl Oncol. 2011:4:157-9.

20. Couture F, D'Anjou F, Desjardins R, Boudreau F, Day R. Role of proprotein convertases in prostate cancer progression. Neoplasia. 2012;14:1032-6.

21. Levesque C, Couture F, Kwiatkowska A, Desjardins R, Guerin B, Neugebauer WA, et al. PACE4 inhibitors and their peptidomimetic analogs block prostate cancer tumor progression through quiescence induction increased apoptosis and impaired neovascularisation. Oncotarget. 2015;6:3680-93.

22. Uhlén $\mathrm{M}$, et al. Tissue-based map of the human proteome. Science. 2015; DOl: https://doi.org/10.1126/science.1260419.

23. Seidah NG, Chrétien M, Day R. The family of subtilisin/kexin like pro-protein and pro-hormone convertases: divergent or shared functions. Biochimie. 1994;76(3-4):197-209.

24. Beaudenon-Huibregtse S, Alexander EK, Guttler RB, Hershman JM, Babu V, Blevins TC, Moore P, Andruss B, Labourier E. Centralized molecular testing for oncogenic gene mutations complements the local cytopathologic diagnosis of thyroid nodules. Thyroid. 2014;24(10):1479-87.

25. Nikiforov YE, Ohori NP, Hodak SP, Carty SE, LeBeau SO, Ferris RL, Yip L, Seethala RR, Tublin ME, Stang MT, Coyne C, Johnson JT, Stewart AF, Nikiforova MN. Impact of mutational testing on the diagnosis and management of patients with cytologically indeterminate thyroid nodules: a prospective analysis of 1056 FNA samples. J Clin Endocrinol Metab. 2011; 96(11):3390-7.

26. Yip L. Molecular markers for thyroid Cancer diagnosis, prognosis, and targeted therapy. J Surg Oncol. 2015;111:43-5.

27. Nikiforova MN, Lynch RA, Biddinger PW, Alexander EK, Dorn GW 2nd, Tallini G, Kroll TG, Nikiforov YE. RAS point mutations and PAX8-PPAR gamma rearrangement in thyroid tumors: evidence for distinct molecular pathways in thyroid follicular carcinoma. J Clin Endocrinol Metab. 2003;88(5):2318-26.

28. Nour N, Mayer G, Mort JS, Salvas A, Mbikay M, Morrison CJ, Overall CM, Seidah NG. The cysteine-rich domain of the secreted proprotein convertases PC5A and PACE4 functions as a cell surface anchor and interacts with tissue inhibitors of metalloproteinases. Mol Biol Cell. 2005; 16(11):5215-26

29. Rodríguez-Rodero S, Fernández AF, Fernández-Morera JL, Castro-Santos P, Bayon GF, Ferrero C, Urdinguio RG, Gonzalez-Marquez R, Suarez C, Fernández-Vega I, Fresno Forcelledo MF, Martínez-Camblor P, Mancikova V, Castelblanco E, Perez M, Marrón PI, Mendiola M, Hardisson D, Santisteban P, Riesco-Eizaguirre G, Matías-Guiu X, Carnero A, Robledo M, Delgado-Álvarez E, Menéndez-Torre E, Fraga MF. DNA methylation signatures identify biologically distinct thyroid cancer subtypes. J Clin Endocrinol Metab. 2013; 98(7):2811-21

30. Levesque C, Fugère $M$, Kwiatkowska A, Couture F, Desjardins R, Routhier $S$, Moussette P, Prahl A, Lammek B, Appel JR, Houghten RA, D'Anjou F, Dory $Y L$, Neugebauer N, Day R. The multi-Leu peptide inhibitor discriminates between PACE4 and Furin and exhibits Antiproliferative effects on prostate Cancer cells. J Med Chem. 2012;55(23):10501-11.

Ready to submit your research? Choose BMC and benefit from:

- fast, convenient online submission

- thorough peer review by experienced researchers in your field

- rapid publication on acceptance

- support for research data, including large and complex data types

- gold Open Access which fosters wider collaboration and increased citations

- maximum visibility for your research: over $100 \mathrm{M}$ website views per year

At $\mathrm{BMC}$, research is always in progress.

Learn more biomedcentral.com/submission 